\title{
When Time is of the Essence: A Rationale for 'Earlier’ Early Intervention
}

\author{
Paula M Barrett ${ }^{1-3 *}$, Marita Cooper ${ }^{3}$ and Anthony B H Teoh ${ }^{3}$ \\ ${ }^{1}$ University of Queensland, Brisbane, St Lucia QLD 4072, Australia \\ ${ }^{2}$ Australian National University, Canberra ACT 0200, Australia \\ ${ }^{3}$ Pathways Health and Research Centre, PO Box 5699, West End, QLD 410, Australia
}

\begin{abstract}
Retrospective reports have consistently demonstrated that many psychiatric disorders have their onset in childhood and adolescence. Childhood onset symptomology typically exhibits a chronic, unremitting course and is related to adverse social, psychological and physical health outcomes. Whilst treatment programs in adulthood aim to reconstruct dysfunctional patterns of behaviors established and maintained over years of experience, early childhood interventions promote healthy beliefs at an age where individuals are more adaptable. This article will provide a rationale for the development and promotion of prevention programs for preschool-aged children by reviewing the prevalence and outcomes of early onset symptomology. This will be followed by an overview of the factors required to develop a framework for prevention programs including risk factors and strengths of preschool-aged children, levels of prevention, and intervention settings. Three commonly utilized treatment approaches will be reviewed prior to the introduction of a proposed model of prevention for working with preschool-aged children. The article concludes with discussion of challenges in prevention research and future directions.
\end{abstract}

Keywords: Early childhood; Prevention; Early intervention; Childhood mental health

\section{Introduction}

It is estimated that approximately 650 million people worldwide are suffering from a psychiatric disorder at any one point in time [1]. Unfortunately, research indicates that approximately $90 \%$ of individuals experiencing clinically significant mental health concerns do not seek treatment, with average delays in individuals seeking intervention exceeding 15 years following symptom onset [2]. Estimates of the lifetime costs in the United States of childhood mental health difficulties specifically are approximately USD \$2 trillion due to poorer academic and vocational outcomes [3]. Compared with treatment programs, typically implemented after significant distress or impairment, prevention programs can reduce the incidence of mental health difficulties prior to onset. Prevalence rates internationally indicate approximately one in five adolescents experience significant mental health difficulties and researchers agree that symptoms are now occurring at increasingly younger ages [4]. This article will provide an overview of childhood mental health literature, with a focus on the prevalence, outcomes and risk factors for early onset psychological difficulties to provide a rationale for early intervention programs. This is followed by discussion of prevention methodology and a review of current prevention programs evaluated in preschool populations to build a model for effective prevention in preschool populations and highlight future directions for prevention research.

\section{Prevalence and Outcomes of Childhood Mental Health Difficulties}

Retrospective reports have consistently demonstrated that many psychiatric disorders have their onset in childhood and adolescence. Prevalence studies in preschool years are rare, potentially due to concerns about diagnostic integrity at this age or the expectation that difficulties are transient. However, a recent study in Norway found that $12.5 \%$ of 4 -year olds met criteria for an emotional or behavioral disorder, a number conservative when compared with international estimates [5-9]. Whilst the appearance of childhood symptoms is not required for the majority of adult psychiatric diagnoses, it does often represent the forewarning of poorer social, psychological and biological outcomes.
Once maladaptive emotional and behavioral patterns develop, they often lead to significant impairment and, when untreated, typically exhibit a chronic and unremitting course $[10,11]$. The specific trajectory of these symptoms, however, is not so clear. Both homotypic and heterotypic continuity of internalizing and externalizing symptoms have been found in preschool-aged children exhibiting clinically relevant difficulties [12]. In a longitudinal study conducted in Denmark, Slemming et al. [13] followed 1,379 children from approximately 7 months of age to 10-12 years. Results demonstrated an association between both anxious-fearful and hostile-aggressive behaviors in preschool-aged children with emotional difficulties at 10-12 years of age. Additionally, preschoolers exhibiting these behaviors demonstrated double the risk of future difficulties than normally adjusted preschoolaged children. Although these results indicate some stability in mental health difficulties, it is unclear whether these findings indicate a persistent disorder in children or are caused by ongoing stressful life events.

Regardless of the type of difficulties experienced, it is commonly accepted that early onset symptomology impacts on an individual's, and frequently a family's, biological, psychological and social outcomes. Disorders with onset in childhood have demonstrated associations with an increased risk for substance use, physical health problems, such as asthma and obesity, comorbid childhood diagnoses, adult psychiatric disorders, and personality disorders [14-16]. In their combined analysis, utilizing three longitudinal epidemiological studies comprising a total of 3,722 participants, Copeland et al. [17] found that diagnosis in childhood led to a three-fold risk of adulthood diagnosis.

*Corresponding author: Paula M Barrett, Pathways Health and Research Centre, PO Box 5699, West End, QLD 4101, Australia, Tel: (07) 33916866 ; E-mail: pbarrett@pathwayshrc.com.au

Received July 11, 2014; Accepted November 07, 2014; Published November 14 2014

Citation: Barrett PM, Cooper M, Teoh ABH (2014) When Time is of the Essence: A Rationale for 'Earlier' Early Intervention. J Psychol Abnorm Child 3: 133. doi:10.4172/2329-9525.1000133

Copyright: (c) 2014 Barrett PM, et al. This is an open-access article distributed under the terms of the Creative Commons Attribution License, which permits unrestricted use, distribution, and reproduction in any medium, provided the original author and source are credited. 
Although homotypic continuity was more frequent, particularly the association of childhood/adolescent to adult behavioral and substance disorders, heterotypic disorders were also seen. As noted earlier, this potentially indicates a stable risk factor in individuals with mental health problems, whether due to genetic risk, impaired psychological coping, or continuing environmental stressors.

Another explanation for stability of psychological symptoms may be variations in brain development in individuals with childhoodonset symptomology. Both longitudinal observations and retrospective studies have noted biological differences between children with early onset symptomology and typically functioning children [1820]. Utilizing fMRI, Gaffrey et al. [19] found significant differences in functional connections for children with preschool-onset major depressive disorder when compared with healthy controls. The regions implicated in this study indicated decreased cognitive control and behavioral flexibility as well as increased self-focused operations in the depressed group. Similar results were found in Fitzgerald et al. [18] study of individuals with Obsessive Compulsive Disorder aged 8-50 years, who demonstrated decreased connectivity in areas responsible for cognitive control and emotional processing. Remarkably, within the atypical group, children with greater symptom severity demonstrated significantly reduced connectivity in the specific cortex associated with cognitive control, when compared with less severe individuals. As well as functional connectivity studies, it has also been reported that highrisk infants can be differentiated from normally developing infants based upon EEG patterns and stress-hormone reactivity [20].

As well as biological and psychological impacts of early onset symptomology, young children exhibiting internalizing or disruptive and aggressive behaviors are likely to experience adverse social outcomes. Young children experiencing mental health problems may experience difficulty mastering developmental competencies and meeting societal expectations. In early childhood, this may appear as classroom disruptions, conduct problems or temper tantrums; however, as children progress into adolescence and adulthood, these difficulties are associated with school dropout, academic and vocational difficulties, peer problems and antisocial behaviors [16,21].

\section{Risk Factors for Childhood Mental Health Difficulties}

The etiology of mental health issues is commonly complex and can often involve not one but a chain of genetic, environmental, social and psychological risk factors. Understanding these risk factors is essential to being able to identify children at risk for developing future mental health difficulties, planning appropriate timing of an intervention and ascertaining necessary treatment components for any intervention. Risk factors may be individual, familial and environmental characteristics that increase the likelihood of adverse developmental outcomes. Although no single risk factor is either necessary or sufficient in the development of a specific disorder, the commonly indicated risk factors in young children are parental psychopathology, temperament and prior symptomology.

Parental psychopathology is commonly accepted as a risk factor for both genetic and environmental pathways towards childhood mental health problems. Family aggregation studies of both the offspring of clinically diagnosed parents and parents of children with mental health difficulties have demonstrated the heritability of mental health issues (for a review of internalizing disorders see [22]). Connell and Goodman's [23] meta-analytic review reviewed associations between parental psychopathology with both internalizing and externalizing disorders in children. From the 230 articles found on parental mental health concerns and childhood internalizing difficulties, it was found that both maternal and paternal psychopathology significantly predicted childhood symptomology [23]. It should be noted, however, that weighted mean effect sizes found from this study were small, and it was found that effects were moderated by child factors, including age and gender, as well as type of parental diagnosis. These findings may indicate the relationship between parental psychopathology and child psychopathology may be more than a direct association with the interaction of parental influences and child influences most likely to determine future symptomology.

Amongst traits genetically implicated in the trajectory of mental health difficulties, temperament is one of the most genetically stable $[24,25]$. During preschoolyears, two temperament types most commonly implicated in mental health trajectories are behavioral inhibition and behavioral disinhibition. These two temperaments exhibit distinct physiological and behavioral responsivity in situations at extreme ends of an approach/avoidance continuum. Behavioral inhibition is characterized by heightened behavioral and emotional reactions to novel or unfamiliar stimuli [26]. Present in approximately one in six preschool-aged children, behavioral inhibition is commonly regarded as one of the earliest identifiable risk factors for future internalizing symptomology [27]. Contrastingly, behavioral disinhibition is defined as a temperamental trait observed as an inability to control or inhibit impulses and behaviors that may be socially inappropriate or involve substantial risk of harm [28]. Research has consistently demonstrated associations between behavioral disinhibition with future externalizing diagnoses such as Attention Deficity/Hyperactivity Disorder, Conduct Disorder, Oppositional Defiant Disorder, and Substance Use Disorders [29]. Despite the clear value in utilizing temperament as an early marker for future psychopathology, researchers are conflicted as to whether these temperamental characteristics are distinct from prodromal symptomology [6].

If temperament can be regarded a personality characteristic, as opposed to psychopathological manifestations, the early signs of a disorder would then be early-onset mental health symptomology. It is possible that early-onset symptomology represents a predisposition to specific symptomology manifesting as different disorders across the lifespan, for example separation anxiety and generalized anxiety in childhood followed by depression in adolescence. Alternatively, early symptomology may indicate a maladaptive pattern of coping progressing from childhood to adulthood leading to future impairment. Regardless of the conceptualization of early-onset symptoms, identification of symptomology allows for individuals to access services promoting adaptive coping skills to both reduce current difficulties and prevent future impairment.

\section{Early Intervention: The Importance of Timing}

Optimal timing is essential in ensuring the effective delivery of a preventative intervention. Such timing can refer to either the chronological timing of the intervention or timing in regards to the onset of symptomology. Chronologically early intervention refers to interventions delivered in childhood years; whereas, symptomatically early intervention can be delivered at any age, simply referring to its placement on the prevention-treatment continuum. This section will be referring to early intervention in the chronological sense, specifically during early childhood - the period from infancy to eight years. Specifically, it will highlight advantages during this period including avoidance of negative outcomes, parental engagement, neuroplasticity, and malleable behavior.

Traditional theories suggesting that mental health disorders did not affect preadolescent children have long been defunct. As highlighted 
earlier, not only do children as young as six years of age experience clinically relevant symptomology, they also meet full 'adult' diagnostic criteria for psychiatric disorders [20]. Furthermore, symptoms that begin in early childhood are likely to lead to school dropout, poorer physical health, a higher likelihood of comorbid diagnoses, and more chronic mental health problems $[15,16,21]$.

Prevention of maladaptive outcomes is not the only goal of prevention programs, with evidence also demonstrating that early interventions can promote protective factors such as enhanced selfesteem and positive future outlook in participants [30]. Delivery of prevention programs in preschool-aged children, in particular, can capitalize on positive experiences, critical periods of development, and have multiple advantages over working with older children, adolescents, and adults including heightened parental involvement. During early childhood, caregivers play a pivotal role in a child's socioemotional skills [31]. The development of positive attachments with primary caregivers is a fundamental milestone for future affective, cognitive and behavioral development. Stable, secure attachments enable children to feel comfortable, viewing the world as a safe and predictable place whereas disorganized or insecure attachments are related to feelings of general mistrust, abandonment and heightened threat perception in their relationships with others and the world. Attachment-based interventions have shown that promoting positive attachments can both improve child wellbeing and behavior as well as overall parental adjustment [32]. Furthermore, provision of effective parenting techniques during early childhood allows for better modeling of appropriate coping behaviors, effective reinforcement strategies, and encourages the adoption of more constructive parenting attitudes.

Throughout an individual's lifespan, environmental forces continually shape the brain through both conscious and unconscious mechanisms. In particular, elements of the brain relevant for social and emotional aspects appear to be affected in response to experiences and events [33]. Early childhood is especially recognized as a highly neuroplastic period in which skills may be more easily attained [20]. Considering these aspects, neuroplasticity in early childhood underlies several benefits of utilizing preventative programs for preschool-aged children. Firstly, due to the brains increased ability for adaptation at this age, individuals are more readily able to obtain new skills. Furthermore, as socio-emotional competence may prevent future negative experiences and increase the likelihood of positive experiences, these environmental factors can further capitalize on neuroplasticity, buffering against maladaptive brain development.

Additionally, children in preschool years are more open and flexible to behavior change. Whilst treatment programs in adulthood aim to reconstruct dysfunctional patterns of behaviors established and maintained over years of experience, early childhood interventions promote healthy beliefs at an age when individuals are more adaptable. Furthermore, programs emphasizing social and emotional skills training allow for children to master these skills before commencing school. Subsequently, children have skills in self-awareness and selfmanagement promoting positive learning outcomes. It is also suggested that teaching these skills in early childhood will increase the likelihood of positive social interactions occurring during the formation of an individual's schemas regarding oneself, others and the world.

Studies comparing intervention effectiveness based on participant age have provided mixed results. In an innovative study by Barrett and colleagues, [34] a sample of 693 children from Grade 6 and Grade 9 completed a universal application of the FRIENDS program. Findings suggested that children in the younger age group reported significantly greater reductions in anxiety and depression compared with the older age group. In particular, children in the younger group with moderate-high levels of anxiety were most responsive to the intervention. In contrast, a recent meta-analysis of child and adolescent anxiety prevention programs found no significant differences for the effectiveness of programs based on participant age [35]. Contrasting results may be due to the small follow-up (approximately half of the included studies had no available follow-up) of studies utilized in Fisak's meta-analysis. Considering that universal prevention programs are delivered at a population level, reductions in symptomology are typically small, with the largest effects commonly found at 12-month follow-up [36]. With consideration of the highlighted advantages of early childhood interventions, further research with long-term followups is required to determine the effect of age on intervention outcomes.

\section{The How and Why of Prevention}

Childhood symptomology has commonly been associated with significant burden for individuals, their families and society. Despite this, the accessibility and volume of child and adolescent mental health services are consistently poorer than for adults [37]. To overcome such challenges when mental health services focus purely on treatment, clinicians and researchers alike have highlighted the importance of prevention and early intervention. Preventative programs focus on enhancing resilience before maladaptive patterns of behavior are established in the course of mental health difficulties. Traditionally, policy makers and funding bodies have been more interested in prevention for targets such as substance abuse and delinquency; outcomes not only more easily measured but also more politically attractive than issues such as anxiety and depression. Despite this, psychological research into prevention programs for childhood mental health has increased exponentially over the past decade.

Treatment interventions have been found to be ineffective in coping with the demand of mental health disorders, with many individuals unable or unwilling to seek empirically supported treatment [38]. Even empirically-based interventions have shown relapse rates as high as $72 \%$ [39]. In contrast, prevention programs afford the opportunity of reducing the overall incidence of mental health disorders prior to the onset of maladaptive patterns of behavior. As well as reducing adverse outcomes, prevention programs have the benefit of simultaneously promoting wellbeing and resiliency in non-clinical populations.

Delivery of prevention programs can be categorized by inclusion criteria for participants on a three-tier basis of Universal, Selected or Indicated prevention. A universal method takes a population delivery approach, where all individuals are included regardless of risk. School settings are a commonly utilized setting for universal prevention programs, with all students of a particular class or year level learning skills proactively. In comparison, both selected and indicated methods target specific individuals in the population. A selected prevention includes individuals or groups on the basis of risk. This may, for example, include individuals from violent communities, indigenous or refugee backgrounds, or offspring of anxious/depressed parents with the aim of reducing the risk of a specific outcome. Finally, indicated prevention includes individuals exhibiting early or mild symptoms of a disorder. Indicated preventions work to treat current maladaptive behaviors, promote effective coping and prevent the further development or worsening of symptoms.

Understandably, each modality has its own strengths and weaknesses. Universal preventions promote a wide range of protective factors in the whole population meaning that all children are afforded the opportunity to build emotional resilience. Furthermore, it has 
been argued that increasing social and emotional skills should provide prophylactic value in that skills will not only support those with current difficulties but also with regular skill rehearsal will encourage positive well-being and prevention of difficulties for nonsymptomatic participants [40]. Despite this, universal programs are costly to implement and research has indicated only small effects/ mixed effectiveness [35,36,41]. Comparatively, both selected and indicated preventative programs are more cost-effective as they are only delivered to the students most likely to require intervention. They also allow for programs to more specifically target risk factors, such as behavioral inhibition, in a selected group or focus on preventing a specific outcome, for example anxious symptomology or substance use. However, a key concern of both clinicians and researchers is the risk of selected and indicated preventative programs stigmatizing children increasing the likelihood of further social isolation, labeling, and peer rejection.

The comparative effectiveness of these three tiers of prevention is currently unclear. In a recent meta-analysis reviewing child and adolescent anxiety prevention programs, regression analysis found no significant difference between effect sizes of universal and targeted prevention programs [35]. In contrast, Calear and Christensen's [41] systematic review of depression prevention programs showed that indicated prevention programs demonstrated greater efficacy than either universal or selective interventions. Further differences were found in Neil and Christensen's [36] systematic review of anxiety prevention programs, which indicated that at post-intervention, universal programs were associated with the highest proportion of significant trials and largest effect size; although, indicated prevention programs were demonstrated most effective at follow-up. These contrasting results may indicate that different levels of prevention are better suited to specific disorders; however, more research is needed to explore whether this was an issue of methodology or true treatment effects.

As well as differing in the inclusion criteria for participants, preventative programs can also be conducted in a range of environments from early years and school settings to clinical and primary care services. The school environment is widely recognized as an ideal setting for prevention programs due to its ability to access a wide range of students in a cost-effective manner [42]. Frequently cited barriers to psychological treatment - including cost, time, availability and location - may be avoided by delivering programs in classrooms $[43,44]$. Additionally, by incorporating social and emotional training into the classroom, students can learn alongside peers and teachers in a supportive setting encouraging daily skill rehearsal and normalization of life challenges [42]. A universal approach to learning social and emotional skills may also hold benefits for destigmatizing mental health issues and intervention. Despite these benefits, there is conflicting research regarding the comparative effectiveness of classroom teachers and trained mental health professionals delivering programs $[35,36,41]$. As such, school-based delivery should include rigorous training of teachers to ensure treatment fidelity and sustainability of the programs.

\section{Components of an Effective Preventative Program}

An efficacious prevention program relies upon the use of an empirically-based framework of risk and protective factors [45]. Whilst risk factors allow for identification of individuals at risk and appropriate intervention timing, protective factors support the construction of intervention targets. In reviewing cross-cultural literature, Bodisch Lynch et al.[46] highlighted the following key protective factors for promoting resilience: autonomy, positive self-concept, realistic and internalized locus of control, empathy, communication and problemsolving skills, self-discipline, and a constructive, positive adult attachment figure. Importantly, these factors were relevant in both enhancing resilience in children at risk as well as improving outcomes for typically developing children [46]. Despite knowledge of these intervention targets, researchers and clinicians employ a range of theoretical approaches as the basis for prevention frameworks.

Researchers and clinicians have, historically, contested the ability of young children to successfully engage in Cognitive Behavioral Therapy (CBT) approaches [47]. Despite CBT's demonstrated effectiveness as a treatment protocol for adolescents and adults, it has been questioned whether young children are capable of meeting CBT's required cognitive demands. However, with the increased use and success of developmentally appropriate play-based methods in the delivery of CBT techniques, these approaches have become the framework of choice as the most frequently cited approach for prevention programs. Christensen et al. [36,41] two systematic reviews of school-based prevention and early intervention programs for depression and anxiety found that $76 \%$ and $78 \%$, respectively, of included studies for children and adolescents used CBT. CBT-based programs commonly incorporate components of psychoeducation, physiological awareness, relaxation training, cognitive restructuring, problem solving, exposure and relapse prevention with the aim of improving an individual's coping strategies in stressful situations as well as their social and emotional competence. Despite the large majority of prevention programs founded in CBT, Christensen et al.[36,41] found that outcome effects were not dependent on intervention type.

Some of the most popular alternative methodologies for working with preschoolers include social skills training, and behavioral approaches. Social skills training protocols may consist of components including behavioral skill rehearsal strategies (role-playing, modeling etc.), social perception strategies, self-regulation, self-monitoring, social problem solving and, parent training [48].These programs hypothesize that poor social skills in childhood and adolescence underlie a range of adverse sequelae for children including peer rejection, behavior problems, academic adjustment and social withdrawal - which are both outcomes and further risk factors in mental health trajectories.

Alternatively, parent-based programs delivered in preschool years and earlier are typically founded in social learning/behavioral theory. These interventions provide parents with training in positive reinforcement, negative reinforcement and consequences to promote desirable child behavior. Commonly utilized parent programs include the well-researched Triple P: Positive Parenting Program [49] and The Incredible Years [50] which promote that assertive discipline is key to encouraging children to take responsibility for their own behavior and gain social awareness. Though interventions focused specifically on improving a child's social and/or emotional competencies may enhance a child's social skills and self-awareness/self-management, they may not alter environmental factors which led to original symptomology such as parent emotional responsivity or parenting style. Comparatively, whilst behaviorally focused parent interventions may reduce environmental reinforcers of internalizing/externalizing behaviors, they may not help children gain emotional regulation skills necessary to prevent internalizing and externalizing difficulties.

\section{Effective Prevention Programs for Preschool-aged Children}

Although still lagging behind the ratio of prevention to treatment articles in medical fields, the past decade has seen a rapid growth in 
psychological prevention literature. Review of current interventions delivered in early childhood found that an overwhelming majority of randomized control trials were targeting children with Autism Spectrum Disorders. This was followed by socio-emotional programs for either offspring of anxious parents or behaviorally inhibited children as well as caregiver-based interventions targeting children with behavioral difficulties. This section will review one program from each of these areas, the Fun FRIENDS anxiety prevention program [51] and Triple P: Positive Parenting Program [49] for child behavior problems, chosen for their particularly well-developed evidence bases as well as ParentChild Interaction Therapy -Emotional Development (PCIT-ED) [52], a relatively new but promising program for preschool depression.

Paula Barrett's FRIENDS programs are one of the most robustly supported protocols for preventing and treating child and adolescent anxiety [53]. The Fun FRIENDS program [51] was developed as a downward extension of the FRIENDS for Life program targeting preschool-aged children. The FRIENDS protocols, although originally an anxiety treatment program, promote themselves as a resilience enhancing intervention that can be used across the continuum from universal prevention to treatment for both preventing internalizing issues and increasing socio-emotional competence. Utilizing playbased cognitive-behavioral strategies, the Fun FRIENDS program is conducted over 10 one-hour sessions in preschool/early years, primary school or clinical setting. A recent randomized controlled trial by Anticich et al. [42] compared the effectiveness of a universal delivery of the Fun FRIENDS program with an active comparison and waitlist control in 488 school children. A particular strength of this study was its use of both asset measures - including behavioral and emotional strength and resilience - as well as deficit measures including behavioral difficulties, behavioral inhibition and anxiety and that researchers also incorporated both parent and teacher report. Results found that whilst both active conditions demonstrated greater reduction in symptomology and improved socio-emotional competence compared with the waitlist group, the Fun FRIENDS intervention was significantly more effective than the active comparison at both postintervention and follow-up.

The Triple P: Positive Parenting Program [49] is a five-level parenting and family support initiative founded in behavioral family intervention. Triple $\mathrm{P}$ incorporates a comprehensive continuum of interventions ranging from a nation-wide television series providing parenting strategies to a 10 -session program with additional homebased training and couple support [54].The overarching aim of the Triple P protocols is to reduce child behavioral problems by modifying familial characteristics maintaining and reinforcing undesirable behavior [49]. A comprehensive meta-analysis of all levels of the Triple P-Positive Parenting Program conducted by Nowak and Heinrichs [55] included 55 studies on the protocols. Results found reliably positive effects for child behavior problems, parenting behavior and parental wellbeing regardless of setting, pre-treatment impairment, and country. Furthermore, studies also demonstrated a tendency for improved quality of the parent relationship.

Developing upon Parent Child Interaction Therapy [56], an established treatment program for preschool-aged children with disruptive disorders, PCIT-ED adds an emotion development module for children with early onset depression. A 14-session program, PCITED utilizes play therapy and behavioral approaches to teach parents behavior management strategies, improve the parent-child relationship, and build emotion regulation skills in the child. The first randomized controlled trial of PCIT-ED was recently published with a sample comprising 54 children aged 3-7 years. All participants met symptom criteria for Major Depressive Disorder according to DSM-IV criteria and were placed in either the PCIT-ED group or a psychoeducation group. Although both groups demonstrated significant improvement at post-intervention in several domains, the PCIT-ED group demonstrated significant improvements across a greater range of domains than the psychoeducation group including internalizing and externalizing symptomology/impairment, emotional development, executive functioning, maternal depression and parenting stress. Additionally, the PCIT-ED group rated significantly greater improvements than the comparison group in emotion recognition, executive functioning, and parenting stress. Despite promising results, this study had heavy attrition rates, approximately $30 \%$ in the PCIT-ED group and over $60 \%$ in the active comparison. Considering this, further studies are required to establish the effectiveness and generalizability of PCIT-ED.

\section{An Integrated Model of Prevention in Preschool Populations}

Utilizing the findings of this paper, the authors have developed a model of prevention for preschool-aged children. Table 1 highlights considerations dependent on the level of prevention utilized, whilst Table 2 provides a proposed framework for an integrated model of prevention in preschool populations.

\section{Challenges in Prevention and Future Research}

Current research into prevention programs is frequently hindered by conflicts over methodology. Firstly, and most importantly, is how to operationalize prevention effectiveness; that is, the method of measuring that a specific outcome, such as psychiatric diagnoses, has not occurred. Researchers have made strong arguments for the use of reduction in current symptomology and impairment, improved resilience and strengths, as well as the prevention of adverse outcomes. As reviewed by Christensen et al. [36,41], the overwhelming majority of studies use a reduction in current symptomology as evidence of effective prevention. Whilst it cannot be denied that lowered current

\section{Levels of prevention}

Universal

- Includes all children regardless of risk

- Ideally delivered in a preschool setting

- Delivered by either teachers, who have completed comprehensive training in both program content and implementation or health professionals

- Promotes protective factors implicated crossdiagnostically (including socio-emotional competence, strong parent and peer attachments, and positive self-concept)

\section{Targeted}

- Includes at risk children such as those exhibiting inhibited/ disinhibited temperament or whose parents have mental health difficulties

- May be delivered in preschool or clinical setting

- Delivered by either teachers, who have completed comprehensive training in both program content and implementation or health professionals

- Promotes overall protective factors as well as targeting specific risk factors related to at-risk group (for example an increased focus on internalizing behaviors in the behaviorally inhibited group)

\section{Indicated}

- Includes children exhibiting early or mild symptomology

- Most likely delivered a clinical setting, although may be delivered by a health professional in a school setting

- Promotes overall protective factors as well as targeting specific risk factors related to current symptomology (for example an increased focus on impulsivity in behaviorally disinhibited group)

Table 1: Intervention recommendations based upon level of prevention. 
Citation: Barrett PM, Cooper M, Teoh ABH (2014) When Time is of the Essence: A Rationale for 'Earlier' Early Intervention. J Psychol Abnorm Child 3: 133. doi:10.4172/2329-9525.1000133

Page 6 of 8

\begin{tabular}{|c|c|c|c|c|c|c|c|c|}
\hline \multicolumn{3}{|c|}{ Components of Prevention Model } & \multicolumn{3}{|c|}{ Treatment targets by component } & \multicolumn{3}{|c|}{ Interventions included by component } \\
\hline $\begin{array}{l}\text { Child-focused } \\
\text { intervention }\end{array}$ & $\begin{array}{l}\text { Parenting skills } \\
\text { intervention }\end{array}$ & $\begin{array}{l}\text { Community- } \\
\text { based } \\
\text { intervention }\end{array}$ & $\begin{array}{l}\text { Child-focused } \\
\text { intervention }\end{array}$ & $\begin{array}{l}\text { Parenting skills } \\
\text { intervention }\end{array}$ & $\begin{array}{l}\text { Community- } \\
\text { based } \\
\text { intervention }\end{array}$ & $\begin{array}{l}\text { Child-focused } \\
\text { intervention }\end{array}$ & $\begin{array}{l}\text { Parenting skills } \\
\text { intervention }\end{array}$ & $\begin{array}{l}\text { Community- } \\
\text { based } \\
\text { intervention }\end{array}$ \\
\hline $\begin{array}{l}\text { Utilization of play- } \\
\text { based and CBT } \\
\text { approaches } \\
\text { Building socio- } \\
\text { emotional } \\
\text { competence and } \\
\text { resilience }\end{array}$ & $\begin{array}{l}\text { Based in social } \\
\text { learning theory } \\
\text { Use of } \\
\text { reinforcement } \\
\text { to promote } \\
\text { desirable } \\
\text { behaviors and } \\
\text { discourage } \\
\text { undesirable } \\
\text { behaviors }\end{array}$ & $\begin{array}{l}\text { Based in } \\
\text { behavioral } \\
\text { approaches } \\
\text { Teachers and } \\
\text { caregivers are } \\
\text { taught resilience } \\
\text { skills for } \\
\text { themselves } \\
\text { Resilience } \\
\text { skills are then } \\
\text { modeled by adult } \\
\text { role models to } \\
\text { children }\end{array}$ & $\begin{array}{l}\text { Increased } \\
\text { autonomy } \\
\text { Positive self- } \\
\text { concept } \\
\text { Assertive } \\
\text { communication } \\
\text { Social awareness } \\
\text { Problem solving } \\
\text { skills } \\
\text { Self-discipline } \\
\text { Self-awareness } \\
\text { Responsible } \\
\text { decision making } \\
\text { Self-management } \\
\text { skills }\end{array}$ & $\begin{array}{l}\text { Reduced use of } \\
\text { coercive/lax/ } \\
\text { authoritarian } \\
\text { parenting } \\
\text { methods } \\
\text { Increased use } \\
\text { of authoritative } \\
\text { parenting } \\
\text { methods } \\
\text { Decreased } \\
\text { adherence } \\
\text { to negative } \\
\text { attribution for } \\
\text { child behavior } \\
\text { Improved } \\
\text { emotional } \\
\text { regulation of } \\
\text { caregivers } \\
\text { Improved } \\
\text { emotional } \\
\text { responsivity of } \\
\text { caregivers }\end{array}$ & $\begin{array}{l} \\
\\
\\
\\
\\
\text { Constructive } \\
\text { positive adult } \\
\text { attachment bonds } \\
\text { with children } \\
\text { Socio-emotionally } \\
\text { competent adult } \\
\text { role models } \\
\text { Community } \\
\text { supportive } \\
\text { of resilience } \\
\text { enhancement } \\
\text { Improved } \\
\text { caregiver } \\
\text { and teacher } \\
\text { adjustment } \\
\end{array}$ & 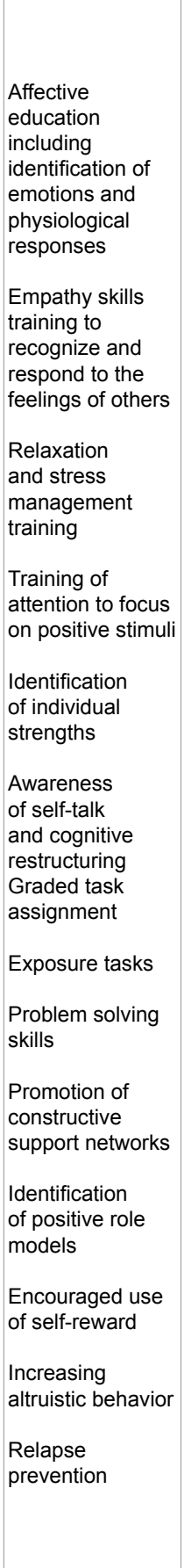 & $\begin{array}{l} \\
\\
\\
\\
\\
\text { Psychoeducation } \\
\text { about factors } \\
\text { contributing to } \\
\text { healthy child } \\
\text { development } \\
\text { Parenting } \\
\text { techniques } \\
\text { (routines, } \\
\text { cohesion, } \\
\text { consistency, } \\
\text { clear instructions, } \\
\text { family rules/ } \\
\text { goals) } \\
\text { Positive parenting } \\
\text { techniques } \\
\text { (descriptive } \\
\text { praise, attention, } \\
\text { rewards) } \\
\text { Behavior } \\
\text { management } \\
\text { strategies } \\
\text { (planned } \\
\text { ignoring, } \\
\text { withdrawal of } \\
\text { privileges, time } \\
\text { out) } \\
\text { Caregiver-child } \\
\text { relationship } \\
\text { quality (family } \\
\text { activities, } \\
\text { caregiver } \\
\text { listening skills) } \\
\\
\end{array}$ & $\begin{array}{l}\text { Affective } \\
\text { education } \\
\text { including } \\
\text { identification of } \\
\text { emotions and } \\
\text { physiological } \\
\text { responses } \\
\text { Empathy skills } \\
\text { training to } \\
\text { recognize and } \\
\text { respond to the } \\
\text { feelings of others } \\
\text { Relaxation } \\
\text { and stress } \\
\text { management } \\
\text { training } \\
\text { Training of } \\
\text { attention to focus } \\
\text { on positive stimuli } \\
\text { Identification } \\
\text { of individual } \\
\text { strengths } \\
\text { Awareness } \\
\text { of self-talk } \\
\text { and cognitive } \\
\text { restructuring } \\
\text { Graded task } \\
\text { assignment } \\
\text { altruistic behavior } \\
\text { Relapse } \\
\text { prevention } \\
\text { Exposure tasks } \\
\text { of self-reward } \\
\text { skills } \\
\text { Problem solving } \\
\text { skills } \\
\text { Promotills training } \\
\text { constructive } \\
\text { support networks } \\
\text { Identification } \\
\text { of positive role } \\
\text { models } \\
\end{array}$ \\
\hline
\end{tabular}

Table 2: Integrated model of prevention for preschool-aged children utilizing three intervention components.

symptomology is associated with a decreased likelihood of future symptomology, this only encompasses one factor in mental health etiology. This method may discount participants still at risk not currently exhibiting symptomology. Utilizing a combination of asset and deficit measures allows for assessment of both risk and protective factors implicated in mental health trajectories, which may offer a more complete view of intervention effects.

A further challenge in early intervention methodology is due to the rapid development that occurs in early childhood. These biological changes affect the interpretation of results with observed changes potentially related to normal development and the resolving of episodic manifestations of developmental challenges. Additionally, early intervention programs may be providing prophylactic effects that require long-term follow-up to become significant. Both of these factors are made further unclear due the lack of longitudinal data collected. As many studies only demonstrate effectiveness during the 
follow-up period, studies of interventions not incorporating follow-up assessment may underestimate treatment effectiveness or overestimate the longevity of improvements [36]. Despite this, longitudinal data requires significant funding and often results in high levels of attrition affecting the generalizability of results. Considering that follow-ups are integral to the accurate evaluation of interventions, researchers must incorporate minimum 3-month post-assessments and consider methods of combatting attrition.

Future research would benefit from comparing the impact of specific definitions of treatment effectiveness (that is symptom/impairment reductions, increased functioning/strengths, etc.) on social factors such as burden to medical services as well as academic and vocational outcomes. Such research may provide further impetus for policy makers to support early intervention efforts. Additionally, with consideration of the rates of mental health problems in ethnic and economically disadvantaged populations, a disproportionate amount of studies have been conducted with middle-upper class Caucasian families. Other than selective prevention programs specifically developed for parents from low socio-economic backgrounds, the FRIENDS programs is one program that has demonstrated significant support in migrant, nonEnglish speaking, and economically disadvantaged populations (for example see $30,59,60$ ). Finally, future research would benefit from exploring whether prevention approaches that are disorder-specific or based upon broad risk factors differ in their ability to prevent a range of mental health difficulties. Whilst, delivery of a cross-diagnostic approach would be understandably a simplified approach to prevention, it is important to discover whether these approaches provide significant effects.

\section{References}

1. Vos T, Flaxman AD, Naghavi M, Lozano R, Michaud C, et al. (2012) Years lived with disability (YLDs) for 1160 sequelae of 289 diseases and injuries 1990 2010: A systematic analysis for the Global Burden of Disease Study 2010. Lancet 380: 2163-2196.

2. Johnson EM, Coles ME (2013) Failure and delay in treatment-seeking across anxiety disorders. Community Ment Health J 49: 668-674.

3. Smith JP, Smith GC (2010) Long-term economic costs of psychological problems during childhood. Soc Sci Med 71: 110-115

4. Sawyer MG, Miller-Lewose LR, Clark JJ (2007) The mental health of 13-17 year olds in Australia: Findings from the National Survey of Mental Health and Wellbeing. J Youth Adolesc 36: 185-194.

5. Kessler RC, Avenevoli S, McLaughlin KA, Green JG, Lakoma MD, et al. (2012) Lifetime co-morbidity of DSM-IV disorders in the US National Comorbidity Survey Replication Adolescent Supplement (NCS-A). Psychol Med 42: 19972010.

6. Egger HL, Angold A (2006) Common emotional and behavioral disorder in preschool children: Presentation, nosology, and epidemiology. J Child Psychol Psychiatry 47: 331-337.

7. Egger HL, Erkanli A, Keeler G, Potts E, Walter BK, et al. (2006) Test-retest reliability of the Preschool Age Psychiatric Assessment (PAPA). J Am Acad Child Adolesc Psychiatry 45: 538-549.

8. Lavigne JV, LeBailly SA, Hopkins J, Gouze KR, Binns HJ (2009) The prevalence of ADHD, ODD, depression, and anxiety in a community sample of 4-year-olds. J Clin Child Adolesc Psychol 38: 315-328.

9. Wichstrom L, Berg-Nielsen TS, Angold A, Egger HL, Solheim E, et al. (2012) Prevalence of psychiatric disorders in preschoolers. J Child Psychol Psychiatry 53: $695-705$

10. Kenneson A, Funderburk JS, Maisto, SA (2013) Risk factors for secondary substance use disorders in people with childhood and adolescent-onset bipolar disorder: Opportunities for prevention. Compr Psychiatry: 54, 439-446.

11. Rapee RM, Schniering CA, Hudson JL (2009) Anxiety disorders during childhood and adolescence: Origins and Treatment. Annu Rev Clin Psycho 5:311-341.
2. Beyer T, Postert C, Muller JM, Furtniss T (2012) Prognosis and continuity of child mental health problems from preschool to primary school: Results of four-year longitudinal study. Child Psychiatry Hum Dev 43: 533-543.

13. Slemming K, Sorenson MJ, Thomsen PH, Obel C, Henriksen TB, et al. (2010) The association between preschool behavioural problems and internalizing difficulties at age 10-12 years. Eur Child Adolesc Psychiatry 19: 787-795.

14. Chang Z, Lichtenstein P, Larsson $H$ (2012) The effects of childhood ADHD symptoms on early-onset substance use: A Swedish twin study. J of Abnorm Child Psychol 40: 425-435.

15. Goodwin RD, Sourander A, Duarte CS, Niemela S, Multimaki P, et al. (2009) Do mental health problems in childhood predict chronic physical conditions among males in early adulthood? Evidence from a community-based prospective study. Psychol Med 39: 301-311.

16. Hill J, Pickles A, Rollinson L, Davies R, Byatt M (2004) Juvenile- versus adultonset depression: Multiple differences imply different pathways. Psychol Med 34: 1483-1493

17. Copeland WE, Adair CE, Smetanin P, Stiff D, Briante C, et al. (2013) Diagnostic transitions from childhood to adolescence to early adulthood. J Child Psychol Psychiatry 54: 791-799.

18. Fitzgerald KD, Welsh RC, Stern ER, Angstadt M Hanna GL, et al. (2011) Developmental alterations of frontal-striatal-thalamic connectivity in obsessivecompulsive disorder. J Am Acad Child Adolesc Psychiatry 50: 938-951.

19. Gaffrey MS, Luby JL, Repovs G, Belden AC, Botteron KN, et al. (2010) Subgenual cingulate connectivity in children with a history of preschooldepression. Neuroreport 21: 1182-1188.

20. Luby JL (2010) Preschool depression: The importance of identification of depression early in development. Curr Dir in Psychol Sci 19: 91-95.

21. Porche MV, Fortuna LR, Lin J, Alegria M (2011) Childhood trauma and psychiatric disorders as correlates of school dropout in a national sample of young adults. Child Dev 82: 982-998.

22. Drake KL, Ginsburg GS (2012) Family factors in the development, treatment, and prevention of childhood anxiety disorders. Clin Child Fam Psychol Rev 15 144-162.

23. Connell AM, Goodman SH (2002) The association between psychopathology in fathers versus mothers and children's internalizing and externalizing behavior problems: A meta-analysis. Psychol Bull 128: 746-773.

24. Fox NA, Henderson HA, Marshall PJ, Nichols KE, Ghera MM (2005) Behaviora inhibition: Linking biology and behavior within a developmental framework. Annu Rev Psychol 56: 235-262.

25. Takahashi $Y$, Yamagata S, Kijima N, Shigemasu K, Ono Y, et al. (2007) Continuity and change in behavioral inhibition and activation systems: A longitudinal behavioral genetic study. Pers Individ Dif 43: 1616-1625.

26. Kagan J, Reznik JS, Snidman N (1987) The physiology and psychology of behavioral inhibition in children. Child Dev 58: 1459-1473.

27. Marysko M, Finke P, Wiebel A, Resch F, Moehler E (2010) Can mothers predict childhood behavioural inhibition in early infancy? Child Adolesc Ment Health 15: 91-96.

28. Muchimba M, Burton M, Yeatman S, Chilungo A, Haberstick BC, et al. (2013) Behavioral disinhibition and sexual risk behavior among adolescents and young adults in Malawi. PLoS ONE 8: e73574.

29. Young SE, Friedman NP, Miyake A, Wilcutt EG, Corley RP, et al. (2009) Behavioral disinhibition: Liability for externalizing spectrum disorders and its genetic and environmental relation to response inhibition across adolescent. J Abnorm Psychol 118: 117-130.

30. Barrett PM, Sonderegger R, Xenos S (2003) Using FRIENDS to combat anxiety and adjustment problems among young migrants to Australia: A national trial. Clin Child Psychol Psychiatry 8: 241-260.

31. McCabe PC, Altamura M (2011) Empirically valid strategies to improve socia and emotional competence of preschool children. Psychol Schools 48: 513540.

32. Aylward P, Murphy P, Colmer K, O'Neill M (2010) Findings from an evaluation of an intervention targeting Australian parents of young children with attachment issues: The 'Through the Looking Glass' (TtLG) project. Aust J Early Child 35: 13-23.

33. Davidson RJ, McEwen BS (2012) Social influences on neuroplasticity: Stress 
and interventions to promote well-being. Nat Neurosci 15: 689-695.

34. Barrett PM, Lock S, Farrell LJ (2005) Developmental differences in universal preventive intervention for child anxiety. Clin Child Psychol Psychiatry 10: 539555.

35. Fisak Jr. BJ, Richard D, Mann A (2011) The prevention of child and adolescent anxiety: A meta-analytic review. Prev Sci 12: 255-268.

36. Neil AJ, Christensen H (2009) Efficacy and effectiveness of school-based prevention and early intervention programs for anxiety. Clin Psychol Rev 29: 208-215

37. Levav I, Jacobsson L, Tsiantis J, Kolaitis G, Ponizovsky A (2004) Psychiatric services and training for children and adolescents in Europe: Results of a country survey. Eur Child Adolesc Psychiatry 13: 395-401.

38. Essau CA (2005) Frequency and patterns of mental health services utilization among adolescents with anxiety and depressive disorders. Depress Anxiety 22: 130-137.

39. Bockting CLH, Schene AH, Spinhover P, Koeter MWJ, Wouters LF, et al. (2005) Preventing relapse/recurrence in recurrent depression with cognitive therapy: a randomized controlled trial. J Consult Clin Psychol 73: 647-657.

40. Greenberg MT (2000) Promoting resilience in children and youth: Preventative interventions and their interface with neuroscience. Ann N Y Acad Sci 1094: 139-150

41. Calear AC, Christensen $\mathrm{H}$ (2010) Systematic review of school-based prevention and early intervention programs for depression. J Adolesc 33: 429-438.

42. Anticich SJ, Barrett PM, Silverman W, Lacherez P, Gillies R (2013) The prevention of childhood anxiety and promotion of resilience among preschoolaged children: A universal school based trial. Adv Sch Ment Health Promot 6 93-21.

43. Barrett PM, Pahl KM (2006) School based intervention: Examining a universal approach to anxiety management. Aust J Guid Couns 16: 55-75.

44. Jorm AF, Wright A (2007) Beliefs of young people and their parents about the effectiveness of interventions for mental disorders. Aust N Z J Psychiatry 41 656-666

45. Giesen F, Searle A, Sawyer M (2007) Identifying and implementing prevention programmes for childhood mental health problems. J Paediatr Child Health 43: 785-789.

46. Bodisch Lynch K, Geller SR, Schmidt MG (2004) Multi-year evaluation of the effectiveness of a resilience-based prevention program for young children. $J$ Prim Prev 24: 335-353.

47. Grave J, Blissett J (2004) Is cognitive behavior therapy developmentally appropriate for young children? A critical review of the evidence. Clin Psychol Rev 24: 399-420.

48. Spence SH (2003) Social skills training with children and young people: Theory, evidence and practice. Child Adolesc Ment Health 8: 84-96.

49. Sanders MR (1999) Triple P - Positive parenting program: Towards an empirically validated multilevel parenting and family support strategy for the prevention of behavior and emotional problems in children. Clin Child Fam Psychol Rev 2: 71-90.
50. Webster-Stratton C (1999) The Incredible Years. BASIC. The parent and children series. A comprehensive course (revised). The Incredible Years, Inc. Seattle, WA

51. Barrett PM (2007) The teaching and training manual for group leaders. Fun Friends Publishing, Brisbane.

52. Stalets MM, Pautsch J, McGrath M, Luby J. Parent-child interaction therapyemotion development. Washington University School of Medicine. (Unpublished manuscript)

53. National Council for Special Education (2011) Evidence of best practice models and outcomes of children with emotional disturbance/behavioural difficulties: An international review (NCSE Research Report No: 7), Meath, Ireland.

54. Fletcher R, Freeman E, \& Matthey S (2011) The impact of behavioral paren training on fathers' parenting: A meta-analysis of the Triple P-Positive Parenting Program. Fathering 9: 291-312.

55. Nowak C, Heinrichs N (2008) A comprehensive meta-analysis of Triple P-Positive Parenting Program using hierarchical linear modeling: Effectiveness and moderating variables. Clin Child Fam Psychol Rev 11: 114-144.

56. Brinkmeyer M, Eyberg SM (2003) Parent-child interaction therapy for oppositional children. In: Kazdin AE, Weisz J. (eds.), Evidence based Psychotherapies for Children and Adolescents. Guildford Press, NY. 